\title{
A branch-and-cut algorithm for the Team Orienteering Problem
}

\author{
Nicola Bianchessi*a, Renata Mansini ${ }^{\mathrm{b}}$ and M. Grazia Speranza ${ }^{\mathrm{c}}$ \\ ${ }^{\mathrm{a}}$ Chair of Logistics Management, Gutenberg School of Management and Economics \\ Johannes Gutenberg University Mainz, Jakob-Welder-Weg 9, D-55128 Mainz, Germany \\ Email: nbianche@uni-mainz.de \\ ${ }^{\mathrm{b}}$ Department of Information Engineering, \\ University of Brescia, via Branze 38, 25123 Brescia, Italy \\ Email: renata.mansini@unibs.it \\ ${ }^{\mathrm{c}}$ Department of Economics and Management, \\ University of Brescia, C.da S. Chiara 50, 25122 Brescia, Italy \\ Email: grazia.speranza@unibs.it
}

\begin{abstract}
The Team Orienteering Problem (TOP) aims at maximizing the total amount of profit collected by a fleet of vehicles while not exceeding a predefined travel time limit on each vehicle. In the last years, several exact methods based on different mathematical formulations were proposed. In this paper, we present a new two-index formulation with a polynomial number of variables and constraints. This compact formulation, reinforced by connectivity constraints, was solved by means of a branch-and-cut algorithm. The total number of instances solved to optimality is 327 out of 387 benchmark instances, 26 more than any previous method. Moreover, 24 not previously solved instances were closed to optimality.
\end{abstract}

Keywords Team Orienteering Problem; two-index mathematical formulation; branch-and-cut algorithm.

\footnotetext{
${ }^{*}$ Corresponding author.
} 


\section{Introduction}

The Team Orienteering Problem (TOP) can be defined over a complete directed graph $G=(V, A)$ with node set $V=\{0, \ldots, n+1\}$ and arc set $A=\{(i, j)$ : $i \neq n+1 ; j \neq 0 ; i \neq j ; i, j \in V\}$. The node set $V$ contains nodes 0 and $n+1$, representing the initial and final depots, respectively, and the set $N=\{1, \ldots, n\}$, indicating the $n$ customers. Each node $i \in V$ has a profit $p_{i}$, with $p_{0}=p_{n+1}=0$. A non-negative travel time $t_{i j}$, with $t_{0, n+1}=0$, is associated with each $\operatorname{arc}(i, j) \in A$. Travel times satisfy the triangle inequality. A fleet $F$ of $m$ identical vehicles is available to serve the customers. When a customer is visited, the corresponding profit is collected by the vehicle serving the customer. Each customer cannot be visited more than once. Each route is a path from node 0 to node $n+1$ in graph $G$. Moreover, the duration of the route associated with each vehicle cannot exceed a predefined threshold $T^{\max }$. The objective is to find a set of vehicle routes each of which not exceeding the maximum time duration and serving a subset of the customers in such a way that the total collected profit is maximized.

The problem, proposed by Chao et al. (1996), has been recently studied and new solution algorithms, both exact and heuristic, have been proposed. In this paper, we focus on mathematical formulations proposed for the problem and on exact solution approaches. If we exclude some preliminary works by Gueguen (1999) and Butt and Ryan (1999) analyzing variants of the TOP (with time windows and with a heterogeneous fleet of vehicles, respectively), the first exact approach is due to Boussier et al. (2007) where a branch-and-price algorithm was proposed. The method, based on a set packing formulation, has the advantage of being easily adaptable to other vehicle routing problems with profits. In particular, the authors apply it to the TOP and to a variant by considering capacity and time window constraints. The authors tested the method on the 387 benchmark instances proposed by Chao et al. (1996) by solving to optimality 270 instances. In de Aragão et al. (2010) different formulations were proposed. However, they did not present a full implementation of the branching scheme and, thus, reported only partial results. In Dang et al. (2013) the authors solved a three-index formulation with a polynomial number of binary variables and generalized subtour elimination constraints. They introduced a branch-and-cut method based on valid inequalities and a set of dominance properties that includes symmetry breaking conditions on the objective function, lower and upper bounds on solution profit and on the number of customers, and clique cuts based on graph incompatibilities. They were able to solve 29 previously unsolved benchmark instances. More recently, Keshtkaran et al. (2016) proposed a branch-and-price approach where the pricing subproblem is solved by a bounded bidirectional dynamic programming algorithm with decremental state space relaxation featuring a two-phase dominance rule relaxation. The authors were able to solve 301 instances out of 387, by closing 17 previously 
unsolved instances. The most recent exact approach is due to El-Hajj et al. (2016), who proposed the effective use of a linear formulation with a polynomial number of variables. Cutting planes are the core component of the algorithm that closed to optimality 12 previously unsolved benchmark instances.

In this paper, we propose a new two-index formulation with a polynomial number of variables and constraints. This compact formulation was inspired by the formulation proposed by Maffioli and Sciomachen (1997) for the Sequential Ordering Problem (SOP) where jobs characterized by a release date and a due time have to be processed on a single machine. A set-up time was also considered for changing from one job to the other. The SOP consists in finding an ordering of the jobs such that the completion time of the job processed last is minimized. The formulation we propose for the TOP was reinforced by the introduction of connectivity constraints and solved by means of a branch-and-cut algorithm. We compared it with the methods of Boussier et al. (2007), Dang et al. (2013), Keshtkaran et al. (2016) and El-Hajj et al. (2016) on the 387 benchmark instances of Chao et al. (1996). The total number of instances solved to optimality is 311 , when tested on a single thread, and 327 when multiple threads are allowed, 10 and 26 more than any previous method, respectively. Most interestingly, 24 previously unsolved instances were solved to optimality, reducing the number of open instances to 49.

The paper is organized as follows. In Section 2, we describe the mathematical formulation used, and in Section 3, the branch-and-cut approach. Computational results are presented in Section 4.

\section{Mathematical formulation}

We model the problem by means of a two-index commodity flow formulation that makes use of three sets of variables. Binary variables $x_{i j},(i, j) \in A$, and $y_{i}, i \in N$, are equal to 1 if arc $(i, j)$ is traversed and customer $i$ is visited, respectively, whereas continuous variables $z_{i j},(i, j) \in A \backslash\{(0, n+1)\}$, represent the arrival time at vertex $j$ of a vehicle coming from vertex $i$. For each set $S \subseteq N$, let $\delta^{+}(S)=\{(i, j) \in A: i \in S, j \notin S\}$ and $\delta^{-}(S)=\{(i, j) \in A: i \notin S, j \in S\}$ be the set of arcs leaving and entering the set $S$, respectively, with $\delta^{+}(i)=\delta^{+}(\{i\})$ and $\delta^{-}(i)=\delta^{-}(\{i\})$. The model is as follows. 


$$
\begin{aligned}
& \max \sum_{i \in N} p_{i} y_{i} \\
& \sum_{j \in N} x_{0 j}=\sum_{i \in N} x_{i, n+1}=m \\
& \sum_{(j, i) \in \delta^{-}(i)} x_{j i}=\sum_{(i, j) \in \delta^{+}(i)} x_{i j}=y_{i} \quad i \in N \\
& z_{0 j}=t_{0 j} x_{0 j} \quad j \in N \\
& \sum_{(i, j) \in \delta^{+}(i)} z_{i j}-\sum_{(j, i) \in \delta^{-}(i)} z_{j i}=\sum_{(i, j) \in \delta^{+}(i)} t_{i j} x_{i j} \quad i \in N \\
& z_{i j} \leq T_{j, n+1}^{\max } x_{i j} \\
& (i, j) \in A \backslash\{(0, n+1)\} \\
& z_{i j} \geq t_{i j}^{0} x_{i j} \\
& (i, j) \in A \backslash\{(0, n+1)\} \\
& y_{i} \in\{0,1\} \\
& i \in N \\
& x_{i j} \in\{0,1\} \\
& 0 \leq x_{0, n+1} \leq m \text {, }
\end{aligned}
$$

where $t_{i j}^{0}=t_{0 i}+t_{i j}$, with $t_{00}=0$, and $T_{j, n+1}^{\max }=T^{\max }-t_{j, n+1}$, with $t_{n+1, n+1}=0$.

This formulation has a polynomial number of variables and constraints. The objective function (1) calls for the maximization of the collected profit. Constraints (2) and (3) are the degree constraints for the depots and the customer nodes, respectively. In particular, constraints (2) impose that exactly $m$ vehicles have to leave and come back to the depot, whereas constraints (3) impose that a customer node $i$ is entered and leaved exactly once if it is visited (i.e. if $y_{i}=1$ ). Constraints (4) bound the flow originating from the initial depot. Flow conservation constraints (5) update the flow value coming out from each visited customer, while constraints (6) set the time limit on the duration of each route. Constraints (7) impose lower bounds on the values of the $\mathbf{z}$ variables in order to restrict the range of feasible values that these variables can assume in fractional solutions. Note that if $x_{i j}=1$ then $z_{i j}$ denotes the arrival time at node $j$, whereas $x_{i j}=0$ implies $z_{i j}=0$. Finally, (8)-(10) are variable definition constraints.

In order to strengthen the formulation, a further constraint can be imposed on the global duration of the routes:

$$
\sum_{(i, j) \in A \backslash\{(0, n+1)\}} t_{i j} x_{i j} \leq m T^{\max }
$$




\section{Branch-and-cut algorithm}

In this section, we briefly describe the branch-and-cut algorithm used to solve the formulation described in Section 2 with the addition of valid inequality (11).

Although equalities (4) and (5) are sufficient to prevent the generation of subtours and to ensure the connectivity of each route, we decided to further strengthen the problem formulation by adding the following more general type of connectivity constraints (see also Toth and Vigo (2002), p. 15, for the use of these cuts in three-index VRP formulations):

$$
\sum_{(i, j) \in \delta^{+}(S)} x_{i j} \geq y_{h} \quad S \subseteq N,|S| \geq 2, h \in S .
$$

The number of the connectivity constraints (CCs) is exponential in the size of $N$. This excludes the possibility to explicit them through a commercial MILP solver even for small-size instances. The CCs are thus dynamically separated along the branch-and-bound tree.

Let $(\overline{\mathbf{y}}, \overline{\mathbf{x}}, \overline{\mathbf{z}})$ be an optimal fractional solution of (1)-(11). Violated connectivity constraints are identified by means of an exact separation algorithm as follows. We consider the graph $\bar{G}=(V, \bar{A})$ induced by the fractional solution, where arc $(i, j) \in \bar{A}$ if the corresponding $\bar{x}_{i j}$ value is greater than 0 . With each arc $(i, j) \in \bar{A}$ a capacity equal to $\bar{x}_{i j}$ is associated. Then, for each pair $\langle i, n+1\rangle, i \in N$, the max-flow/min-cut problem is solved on graph $\bar{G}$, where vertex $i$ is the source of the flow and vertex $n+1$ is the sink. We consider the partition $S$ induced by the min-cut such that $i \in S$. If $n+1 \notin S$ and the value of the flow is less than $\bar{y}_{v}$ for some $v \in S$, then a violated connectivity constraint has been identified. The violated constraint is inserted for $i=\operatorname{argmax}_{v \in S}\left\{\bar{y}_{v}\right\}$. Max-flow/min-cut problems are solved by means of the algorithm described in Boykov and Kolmogorov (2004).

\section{Experimental results}

The branch-and-cut algorithm was implemented in $\mathrm{C}++$, by using CPLEX 12.6 Concert Technology. The code was compiled in release mode with MS Visual $\mathrm{C}++2012$ Express Edition for Windows Desktop. The experiments were carried out on a 64-bit Windows computer, with the Intel Xeon processor W3680, 3.33 $\mathrm{GHz}$, and $12 \mathrm{~GB}$ of RAM. CPLEX built-in cuts were used in all experiments. We set IloCplex::ParallelMode to 1 in order to force CPLEX to always use deterministic algorithms. Parameter IloCplex::Threads has been set to different values as illustrated in the following. For all the other CPLEX's parameters we kept their default values. All experiments were run on the 387 benchmark instances proposed by Chao et al. (1996) with a time limit of 2 hours as in Boussier et al. (2007), Dang 
Table 1: Benchmark instances

\begin{tabular}{c|rrcr} 
Set & $\#$ & $|N|$ & $m$ & \multicolumn{1}{c}{$T^{\max }$} \\
\hline 1 & 54 & 32 & $2-4$ & $3.8-22.5$ \\
2 & 33 & 21 & $2-4$ & $1.2-42.5$ \\
3 & 60 & 33 & $2-4$ & $3.8-55.0$ \\
4 & 60 & 100 & $2-4$ & $3.8-40.0$ \\
5 & 78 & 66 & $2-4$ & $1.2-65.0$ \\
6 & 42 & 64 & $2-4$ & $5.0-200.0$ \\
7 & 60 & 102 & $2-4$ & $12.5-120.0$
\end{tabular}

et al. (2013), Keshtkaran et al. (2016), and El-Hajj et al. (2016). Although a direct comparison of methods using different hardware is not a straightforward task, we recall here that Boussier et al. (2007) used a PC Pentium IV 3.2 GHz, Dang et al. (2013) tested their branch-and-cut method on an AMD Opteron $2.60 \mathrm{GHz}$ by using CPLEX 12.4 as MIP solver, Keshtkaran et al. (2016) conducted their experiments on a single core of an Intel Core i7 $3.6 \mathrm{GHz}$, and finally El-Hajj et al. (2016) run tests on an AMD Opteron 2.60GHz, by using CPLEX 12.5 as MIP solver.

The data set by Chao et al. (1996) consists of 387 instances divided into 7 sets according to the number of control points (customers) ranging from 21 to 102. In each set the instances differ only for the number of vehicles available to serve the customers (from 2 to 4 ) and for the value of the maximum time duration of a route. For each set, Table 1 reports the number of instances in the set ('\#'), the number of customers in each instance (' $|N|$ '), the range values for the number of available vehicles (' $m$ ') and for the maximum time duration of a route (' $T^{\text {max }}$ ').

As previously done in Dang et al. (2013), a node is removed if the travel time of the tour containing only such a node exceeds the time limit $T^{\max }$. Similarly, we eliminated all the inaccessible arcs.

We first show the results on the effectiveness of the CCs and then compare our method with the methods proposed by Boussier et al. (2007), Dang et al. (2013), Keshtkaran et al. (2016), and El-Hajj et al. (2016). A detailed comparison on the partial set of instances tested in de Aragão et al. (2010) is reported in Keshtkaran et al. (2016).

Table 2 is devoted to testing the effectiveness of the CCs. This first set of experiments was carried out setting the number of threads available to CPLEX to 1 (IloCplex::Threads $:=1$ ). The first three columns report the set of instances ('Set'), the number of vehicles (' $m$ ') and the number of instances in the row ('\#'). Then, the following six columns show the results for the branch-and-cut algorithm on the compact formulation (1)-(11) with the separation of the CCs. The six columns report the number of instances solved to optimality, the average com- 
putational time needed in seconds to find the optimal solution, the number of unsolved instances, the final average optimality gap computed on the unsolved instances, the average number of branch-and-bound nodes inspected and the average number of cuts generated. The last five columns report similar statistics for the case where the CCs are not included. In general, including the CCs is beneficial, in terms of number of instances solved, computational time needed to find the optimal solutions, and optimality gap for the unsolved instances. There are only few cases (see for example the results for Set 5) where the solution of the pure compact formulation gives slightly better results. In such cases, it seems that the separation of the CCs guides the search towards area of the solution space not including good feasible solutions, leading CPLEX to explore a higher number of nodes, and to sometimes miss the optimal solution.

Table 3 summarizes the results of the comparison between our branch-and-cut algorithm with $\mathrm{CCs}$ and the other state-of-the-art exact algorithms. We included in the comparison two versions of our algorithm: single and multi-thread. In the first version, CPLEX is forced to use 1 thread only (IloCplex::Threads $:=1$ ), while in the second one CPLEX can use up to 6 threads (IloCplex::Threads $:=6$ ). For each known method, each row reports the number of instances solved to proven optimality (out of the total number of instances in the set), and the average computational time w.r.t. such instances optimally solved. Since Dang et al. (2013) and El-Hajj et al. (2016) do not report complete results on the computational times, only the number of instances solved to optimality is shown in the table for such methods. For each version of our algorithm we report, in addition to the number of solved instances and their average computational time, the average optimality gap (\%) computed w.r.t the instances not solved to proven optimality. In terms of total number of instances solved, the single thread version solves 10 instances more than each of the other methods, the multi-thread 26 more.

As each method solves different sets of instances, it is also interesting to look at the specific instances solved. Then, in Table 4 we list the instances solved to optimality for the first time by means of our branch-and-cut algorithm. Columns labeled with 'Instance', ' $n$ ', ' $m$ ', and ' $T^{\max }$ ' indicate the name of the instance solved, the number of customers, the number of vehicles and the time limit, respectively. Column 'Opt.' reports the objective function optimal value. The last two columns show the computational time in seconds required to solve the instance in the single-thread and multi-thread versions. A '-' indicates that the instance could not be solved to optimality within the time limit. 


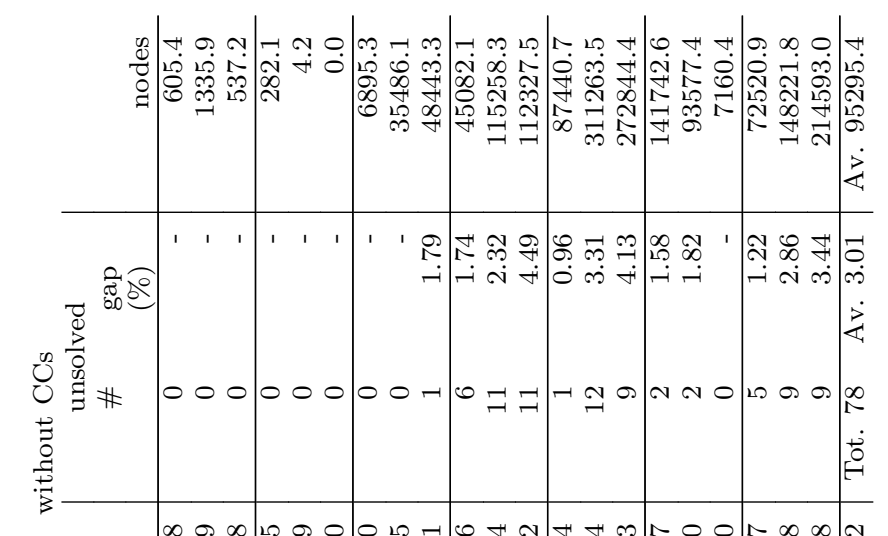

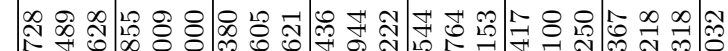
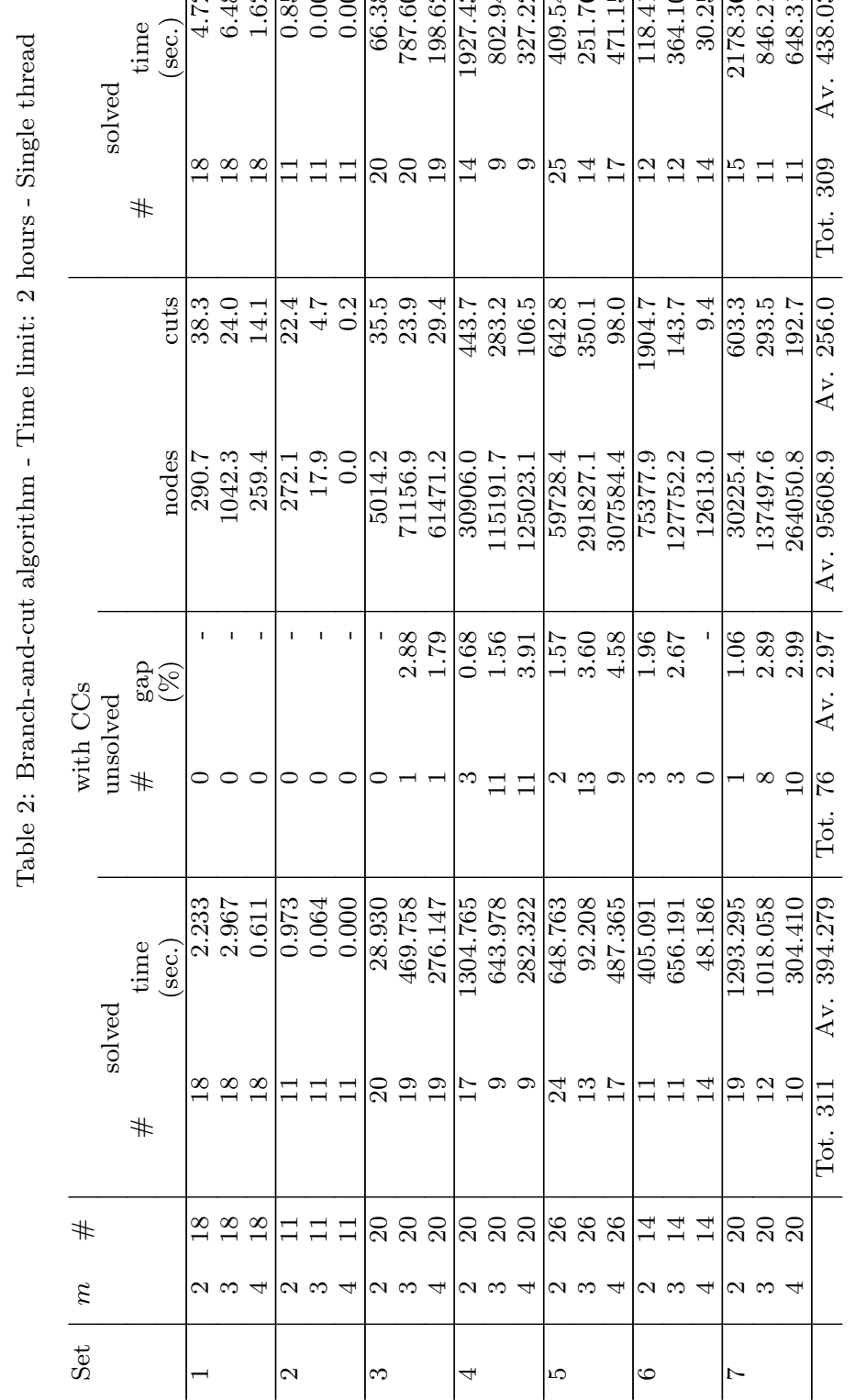

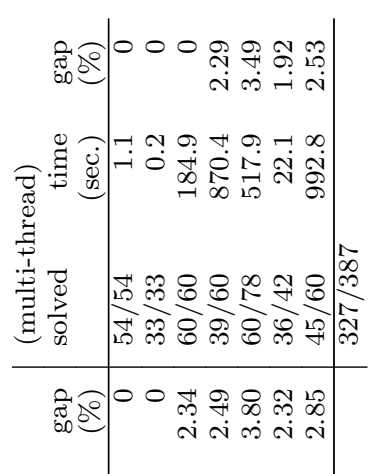

రิ 青焉

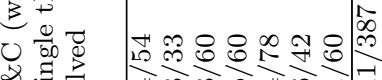

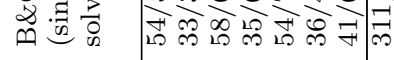

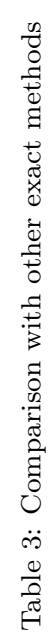

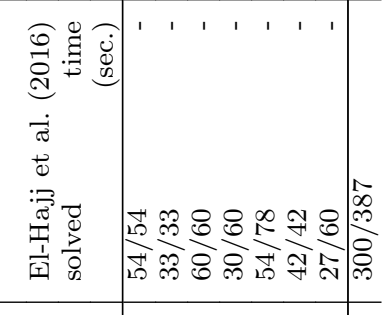

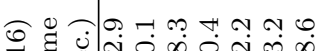

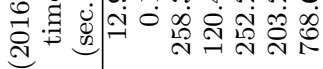
สं

屯

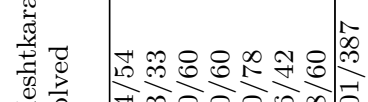
要

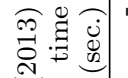
สं

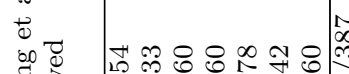

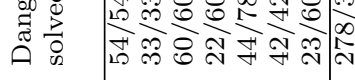

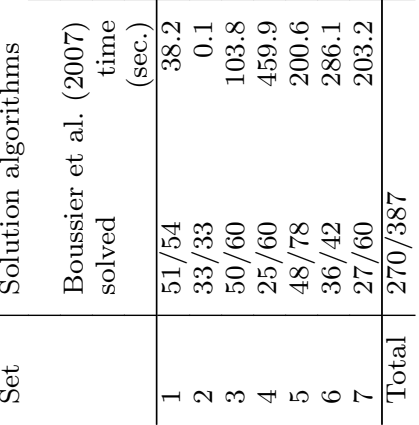


Table 4: New optimal solutions

\begin{tabular}{lrrr|r|rr} 
& & & & \multicolumn{2}{|c}{ Time } \\
Instance & $n$ & $m$ & $T^{\max }$ & Opt. & Single thread & Multi-thread \\
\hline p4.2.c & 76 & 2 & 35 & 452 & 3.8 & 3.0 \\
p4.2.d & 90 & 2 & 40 & 531 & 115.4 & 32.5 \\
p4.2.e & 97 & 2 & 45 & 618 & 83.6 & 38.3 \\
p4.2.g & 98 & 2 & 55 & 757 & 513.3 & 178.7 \\
p4.2.n & 98 & 2 & 90 & 1174 & 885.5 & 289.4 \\
p4.2.o & 98 & 2 & 95 & 1218 & 1528.7 & 546.6 \\
\hline p4.3.d & 45 & 3 & 26.7 & 335 & 5.2 & 2.1 \\
p4.3.e & 56 & 3 & 30 & 468 & 55.4 & 16.4 \\
p4.3.f & 71 & 3 & 33.3 & 579 & 35.1 & 9.0 \\
p4.3.j & 97 & 3 & 46.7 & 861 & - & 4196.8 \\
p4.3.n & 98 & 3 & 60 & 1121 & - & 6633.4 \\
\hline p4.4.g & 49 & 4 & 27.5 & 461 & 33.5 & 9.0 \\
\hline p5.3.r & 64 & 3 & 30 & 1125 & - & 6979.1 \\
\hline p7.2.j & 100 & 2 & 100 & 646 & 1263.0 & 282.0 \\
p7.2.k & 100 & 2 & 110 & 705 & 1387.8 & 390.1 \\
p7.2.1 & 100 & 2 & 120 & 767 & 690.0 & 126.4 \\
p7.2.m & 100 & 2 & 130 & 827 & 2156.1 & 1009.4 \\
p7.2.n & 100 & 2 & 140 & 888 & 1083.4 & 85.1 \\
p7.2.o & 100 & 2 & 150 & 945 & 1615.7 & 627.7 \\
p7.2.p & 100 & 2 & 160 & 1002 & 4234.1 & 3154.0 \\
p7.2.r & 100 & 2 & 180 & 1094 & 3063.1 & 4147.9 \\
p7.2.s & 100 & 2 & 190 & 1136 & 4417.5 & 5789.1 \\
\hline p7.3.o & 100 & 3 & 100 & 874 & - & 5640.8 \\
p7.3.p & 100 & 3 & 106.7 & 929 & & 6231.4
\end{tabular}

\section{Conclusions}

A new compact formulation for the Team Orienteering Problem, with a polynomial number of variables and constraints, was presented. Such a formulation was reinforced with connectivity constraints and solved with a branch-and-cut algorithm. The approach was compared with all the previously proposed exact methods that were tested on the 387 benchmark instances. In terms of total number of instances, we solved to optimality 311 instances on a single thread and 327 on multiple threads. This implies that we solved 10 more instances, with a single thread, and 26 more instances, with multiple threads, than any other previous method. Most interestingly, we solved 24 previously unsolved instances. This is due to the fact that the different methods solve different sets of instances and, somehow, complement each other. The extension of the proposed formulation to other routing problems is a promising research direction. 


\section{References}

S. Boussier, D. Feillet, and M. Gendreau. An exact algorithm for team orienteering problems. 4OR, 5:211-230, 2007.

Y. Boykov and V. Kolmogorov. An experimental comparison of min-cut/max-flow algorithms for energy minimization in vision. In IEEE Transactions on Pattern Analysis and Machine Intelligence (PAMI), 2004.

S.E. Butt and D.M. Ryan. An optimal solution procedure for the multiple tour maximum collection problem using column generation. Computers \& Operations Research, 26(4):427-441, 1999.

I.-M. Chao, B.L. Golden, and E.A. Wasil. The team orienteering problem. European Journal of Operational Research, 88:464-474, 1996.

D.-C. Dang, R. El-Hajj, and A. Moukrim. A branch-and-cut algorithm for solving the team orienteering problem. In C. Gomes and M. Sellmann, editors, Integration of AI and OR Techniques in Constraint Programming for Combinatorial Optimization Problems, Lecture Notes in Computer Science, pages 332-339. Springer Berlin Heidelberg, 2013.

M. Poggi de Aragão, H. Viana, and E. Uchoa. The team orienteering problem: Formulations and branch-cut and price. In OASIcs-OpenAccess Series in Informatics, volume 14. Schloss Dagstuhl-Leibniz-Zentrum fuer Informatik, 2010.

R. El-Hajj, D.-C. Dang, and A. Moukrim. Solving the team orienteering problem with cutting planes. Computers \& Operations Research, 74:21-30, 2016.

C. Gueguen. Méthodes de résolution exacte pour les problémes de tournées de véhicules. $\mathrm{PhD}$ thesis, 1999.

M. Keshtkaran, K. Ziarati, A. Bettinelli, and D. Vigo. Enhanced exact solution methods for the team orienteering problem. International Journal of Production Research, 54:591-601, 2016.

F. Maffioli and A. Sciomachen. A mixed-integer model for solving ordering problems with side constraints. Annals of Operations Research, 69:277-297, 1997.

P. Toth and D. Vigo, editors. The Vehicle Routing Problem. Society for Industrial and Applied Mathematics, Philadelphia, PA, USA, 2002. ISBN 0-89871-498-2. 